\title{
Preparation in the business and practice of medicine: perspectives from recent gynecologic oncology graduates and program directors
}

\author{
Matthew Schlumbrecht ${ }^{1 *}$ (D, John Siemon ${ }^{2}$, Guillermo Morales', Marilyn Huang ${ }^{1}$ and Brian Slomovitz ${ }^{1}$
}

\begin{abstract}
Background: Preparation in the business of medicine is reported to be poor across a number of specialties. No data exist about such preparation in gynecologic oncology training programs. Our objectives were to evaluate current time dedicated to these initiatives, report recent graduate perceptions about personal preparedness, and assess areas where improvements in training can occur.

Methods: Two separate surveys were created and distributed, one to 183 Society of Gynecologic Oncology candidate members and the other to 48 gynecologic oncology fellowship program directors. Candidate member surveys included questions about perceived preparedness for independent research, teaching, job-hunting, insurance, and billing. Program director surveys assessed current and desired time dedicated to the topics asked concurrently on the candidate survey. Statistical analysis was performed using Chi-squared (or Fisher's exact test if appropriate) and logistic regression.

Results: Survey response rates of candidate members and program directors were $28 \%$ and $40 \%$, respectively. Candidate members wanted increased training in all measures except retrospective protocol writing. Female candidates wanted more training on writing letters of intent $(\mathrm{LOI})(p=0.01)$ and billing $(p<0.01)$. Compared to their current schedules, program directors desired more time to teach how to write an investigator initiated trial ( $p=0.01)$. 94\% of program directors reported having career goal discussions with their fellows, while only $72 \%$ of candidate members reported that this occurred $(p=0.05)$.

Conclusion: Recent graduates want more preparation in the non-clinical aspects of their careers. Reconciling program director and fellow desires and increasing communication between the two may serve to achieve the educational goals of each.
\end{abstract}

Keywords: Fellowship education, Program directors, Survey, Gynecologic oncology

\section{Background}

Gynecologic oncology training programs have evolved much over the last ten years, and the volume of information that fellows are required to master has significantly increased. As it is has been reported that matriculating fellows are often ill-equipped for the rigors of a gynecologic oncology fellowship [1], there are increasing pressures on training programs to ensure adequate surgical experiences

\footnotetext{
* Correspondence: mschlumbrecht@miami.edu

'Division of Gynecologic Oncology, Sylvester Comprehensive Cancer Center, University of Miami, 1121 NW 14th St, Suite 345C, Miami, FL 33136, USA

Full list of author information is available at the end of the article
}

while providing thorough didactic teaching in a short amount of time. Education in the non-clinical aspects of medicine, including skills to be a successful academician, experience with trial development, and exposure to different types of practice environments, may be lacking in lieu of basic science and clinical programs. At a time when recent fellowship graduates report dissatisfaction with their fellowship didactic lectures [2], it is important to understand the areas where they perceive weaknesses, and address these concerns in order to work towards further optimization of training programs. Our objective was to evaluate the perceptions of recent fellowship graduates in 
regards to their preparation for the non-clinical responsibilities of medicine, to evaluate current fellowship program education for such non-clinical responsibilities, and to determine if fellowship program directors are themselves satisfied with their own content curricula.

\section{Methods}

Approval was obtained from the University of Miami Institutional Review Board. Two separate de novo surveys were created (Additional files 1 and 2). The first survey was for recent gynecologic oncology fellowship graduates who had not yet passed the oral certification exam in gynecologic oncology. These individuals are candidates for membership in the Society of Gynecologic Oncology (SGO), and were identified by a query of the SGO member database. The candidate survey contained questions about demographics, education history, and current practice setting. Additional questions about perceived preparation for a number of issues in post-training practice were posed in yes/no format, and addressed comfort with grant writing, protocol writing, effective teaching, billing and coding, malpractice insurance and medical-legal issues, financial planning, and disability; follow-up questions about these topics asked whether or not they would have preferred more training on each. Candidate members were also asked about how they obtained their first jobs, including faculty mentorship during the process, use of a headhunter, and contract review.

The second survey was developed for current gynecologic oncology fellowship program directors. As the subspecialty of gynecologic oncology is currently undergoing a transition in oversight from the American Board of Obstetrics and Gynecology (ABOG) to the Accreditation Council for Graduate Medical Education (ACGME), program directors were identified by a query of both organizations. Associate program directors were not included. Length of the program was ascertained. The survey then inquired about current number of didactic hours dedicated to each of the following topics over the length of fellowship training: protocol writing, drafting a grant proposal/letter of intent, being an effective teacher, billing/ coding, medical-legal concerns, financial planning, disability, and the Affordable Care Act. Program directors were then asked how many hours they would prefer to spend on each of these topics.

The surveys for both program directors and candidate members were released via email in January, 2017, with a link to the questionnaire. The survey link was active for six weeks, and three separate email invitations to participate were sent. A statement of implied informed consent was included in the survey instructions. Responses were collected anonymously and stored in a RedCap database.
Statistical analyses were completed using STATA IC (StataCorp, College Station, TX). All data was used, even if the surveys were not completed in toto. Summary statistics were generated to describe the cohort. Chi-square testing (or Fisher's exact when appropriate) was used to analyze proportional associations between groups. Logistic regression was used to estimate associations between binary variables. All tests were two-sided, and $p$-values $<0.05$ were considered statistically significant.

\section{Results}

Of the 183 candidate members contacted, fifty-one (28\%) completed the survey. Demographic characteristics are shown in Table 1 . Seventy one percent $(71 \%)$ of the respondents were female, and the median age was 36 years (range $32-44$ ). The majority had completed their fellowships after 2013, with 75\% attending threeyear programs. Nearly $57 \%$ of participants were in university-based practices, while 33\% reported being employed in community/academic hybrid programs, and $10 \%$ in community based programs.

Ninety-two percent of respondents felt comfortable writing a retrospective protocol and $74 \%$ felt comfortable drafting a letter of intent, but only $26 \%$ reported comfort writing a grant proposal. Even fewer (14\%) felt comfortable writing an investigator initiated therapeutic trial. There were no differences in reported

Table 1 Demographics of candidate member respondents $(n=51)$

\begin{tabular}{lc}
\hline $\mathrm{N}(\%)$ & \\
\hline Gender & $14(27.5)$ \\
Male & $36(70.6)$ \\
Female & $1(1.9)$ \\
Other & \\
Current Age & $15(29.4)$ \\
Less than 35 years & $29(56.9)$ \\
35-39 years & $7(13.7)$ \\
40-44 years & \\
Year of Fellowship Graduation & $3(5.8)$ \\
2008-2010 & $14(27.5)$ \\
2011-2014 & $34(66.7)$ \\
2014-2016 & \\
Length of Fellowship Program & $38(74.5)$ \\
3 years & $13(25.5)$ \\
4 years & \\
Current Practice Setting & $5(9.8)$ \\
Community based & $26(56.9)$ \\
University based & $17(33.3)$ \\
Community/Academic hybrid &
\end{tabular}


comfort levels by gender, age, time since fellowship, or length of fellowship.

Figure 1 shows candidate-reported exposure to educational opportunities and career development during their training. Twenty percent or fewer of respondents had attended a protocol writing workshop $(20 \%)$ or billing and coding courses (2\%), and reported little education in documentation and coding (14\%), ICD-10 transition (14\%), the Affordable Care Act (10\%), malpractice insurance $(20 \%)$, and disability insurance (12\%).

Figure 2 shows areas in which candidate members reported whether or not they received adequate education on non-clinical topics during fellowship. The greatest proportions of candidate members wanted additional education in billing, coding, and documentation (94\%) and the Affordable Care Act (82\%). Topics about which additional education was least desired included retrospective protocol writing (48\%), how to be an effective teacher (64\%), and writing letters of intent (64\%). Despite this, working in an academic practice was highly associated with the desire for more training in writing a letter of intent (OR 3.45 [95\% CI 1.03-11.55], $p=0.04$ ). Academic candidate members were also more likely to want additional training in drafting IIT, though this did not reach statistical significance (OR 2.97 [95\% CI 0.8010.90, $p=0.10)$. Age, length of fellowship, and time since completion of fellowship were not associated with reported interest in more education about any topic queried. Compared with males, a greater proportion of females wanted more teaching on billing and coding (79\% vs. $\left.100 \%, x^{2}=10.37, p=0.005\right)$ and writing letters of intent ( $36 \%$ vs. $\left.74 \%, \chi^{2}=6.40, p=0.01\right)$. Males were less likely to want more training on disability insurance (OR 0.28 [95\% CI 0.07-1.09], $p=0.059$ ) and grant writing (OR 0.30 [95\% CI 0.08-1.10], $p=0.07$ ), though these did not reach statistical significance. Formal education in certain subject areas was negatively associated with a desire for further training on those subjects, including how to be an effective teacher (OR 0.11 [95\% CI 0.03$0.45), p=0.002)$, the Affordable Care Act $(0.03[95 \% \mathrm{CI}$ $0.002-0.34], p=0.004$ ), and disability insurance (OR 0.22 [95\% CI 0.06-0.87], $p=0.03$ ). Of those who did not receive any teaching on the Affordable Care Act, $89 \%$ reported wanting more training.

Of the 48 fellowship program directors contacted, 19 completed the survey for a response rate of $40 \%$. Sixty-five percent of the respondents directed threeyear programs, while $35 \%$ directed four-year programs. The current didactic time for each program dedicated to the non-clinical practice of medicine is reported in Table 2. There were no associations between the length of the program and the amount of didactic time spent on any one topic. Program directors did indicate, however, that they would prefer more didactic time be spent on how to write an investigator-initiated trial compared to the current time allotted in their programs $\left(x^{2}=6.11, p=0.01\right)$.

Table 3 shows candidate member and fellowship director responses to mentorship and job-hunting questions. Compared with fellowship directors, fewer candidate members reported that career goals were discussed $\left(72 \%\right.$ vs. $\left.94 \%, x^{2}=3.89, p=0.05\right)$ and that they received encouragement from their program directors to review employment agreements with a

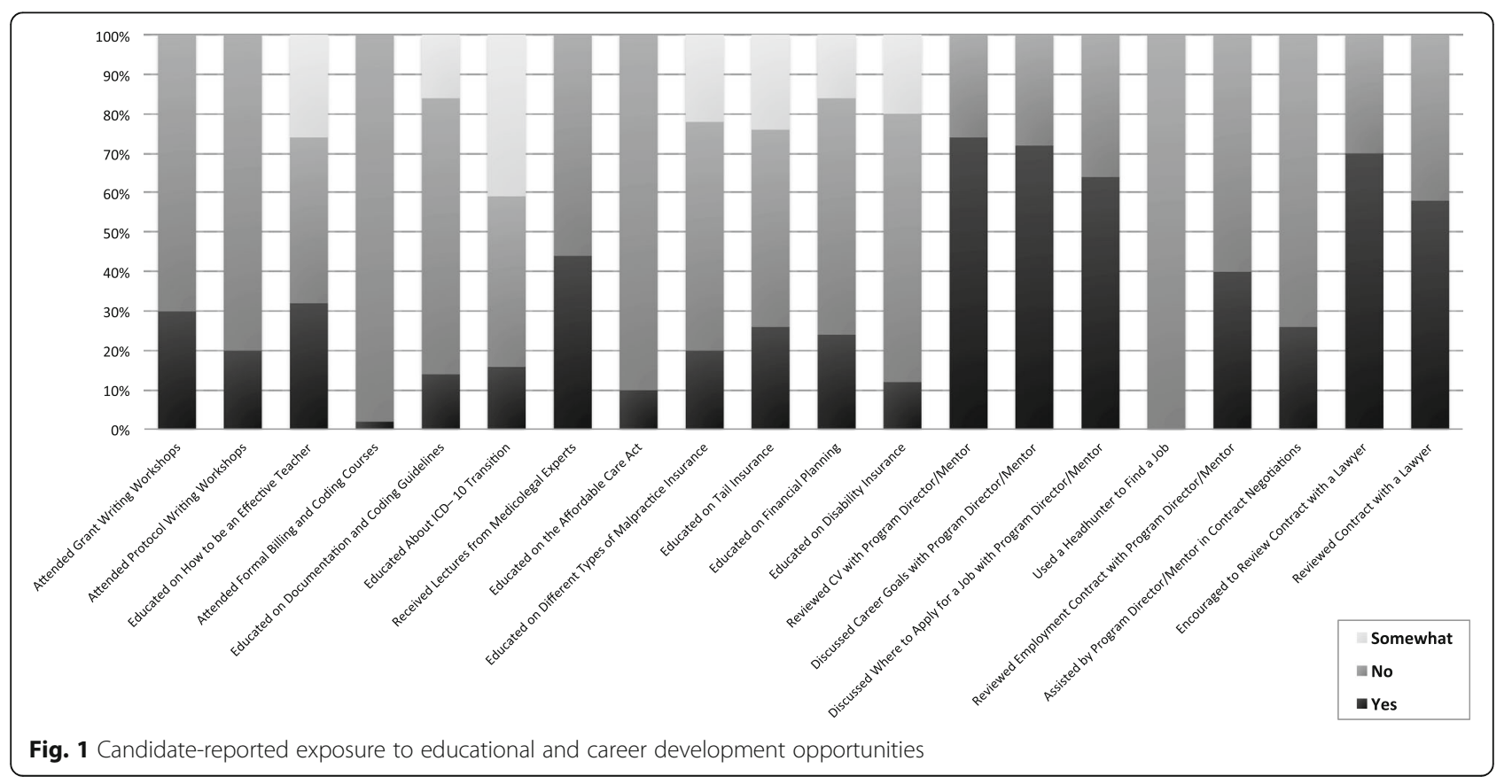




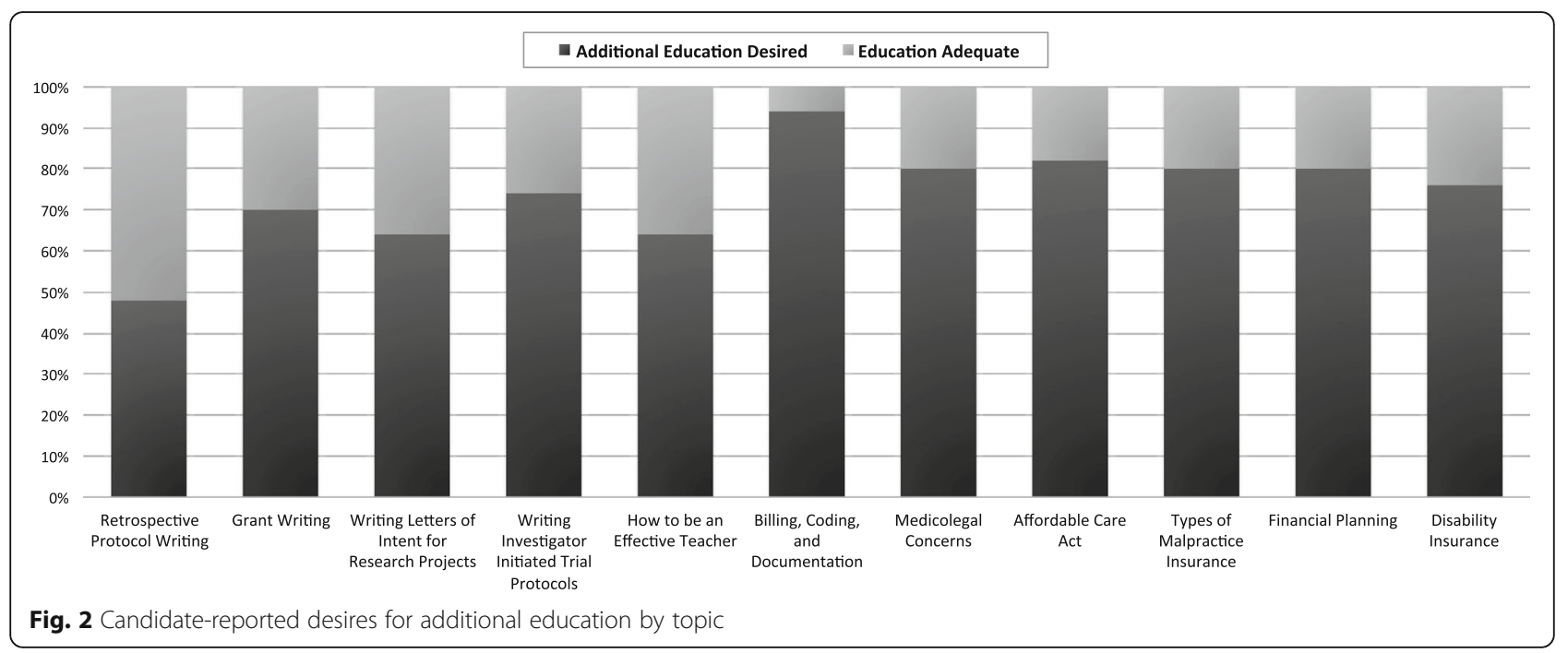

lawyer $\left(70 \%\right.$ vs. $\left.94 \%, x^{2}=4.39, p=0.05\right)$. Notably, those who were encouraged to review their contracts with a lawyer were more likely to actually do so compared with those who did not (OR 6.87 [95\% CI 1.77-26.76], $p=0.005$ ).

\section{Discussion}

With increasing pressure on fellowship programs to train competent gynecologic oncologists in an era of rapidly advancing surgical and chemotherapeutic evolution, it is important to also recognize the concerns of trainees about adequate preparation for the practical aspects of being independent physicians. Our study shows that while recent fellowship graduates indicate a high level of comfort with some aspects of both the academic and business components of post-training employment, there remains a significant disparity in the perceived preparation for many of these important facets of practice.

The findings reported here are not unique to gynecologic oncology. In fact, other surgical specialties have reported a recognition of the importance of training in the business of medicine and a lack of trainee knowledge in this arena. Fakhry et al. [3] published the results of a survey of surgical residents and attending physicians, which included self-assessments of perceived knowledge and expertise in billing, coding, documentation, and insurance reimbursement. While $92 \%$ of residents reported that expertise in documentation and coding would make a difference in their practices, only 54\% of residents accurately answered questions about billing correctly. Nearly $90 \%$ of both residents and faculty, however, thought further education on the topic was a crucial part of residency training. Similarly, a survey of general

Table 2 Program director report of current didactic time versus desired didactic time per topic ( $n=$ number of programs) over the entire length of fellowship

\begin{tabular}{|c|c|c|c|c|c|}
\hline Educational Topic & $\begin{array}{l}\text { Current Time } \\
0-2 \mathrm{~h}(\mathrm{n})\end{array}$ & $\begin{array}{l}\text { Current } \\
\text { Time } \\
\geq 3 \mathrm{~h}(\mathrm{n})\end{array}$ & $\begin{array}{l}\text { Desired Time } \\
0-2 \mathrm{~h}(\mathrm{n})\end{array}$ & $\begin{array}{l}\text { Desired Time } \\
\quad \geq 3 \mathrm{~h}(\mathrm{n})\end{array}$ & $x^{2} p$-value \\
\hline Retrospective protocol writing & 11 & 8 & 7 & 11 & 0.25 \\
\hline Writing letter of intent & 12 & 7 & 7 & 11 & 0.14 \\
\hline Writing grant proposal & 11 & 8 & 6 & 12 & 0.13 \\
\hline Writing investigator initiated trial & 13 & 6 & 5 & 13 & 0.01 \\
\hline Being an effective teacher & 10 & 9 & 5 & 13 & 0.12 \\
\hline Billing, coding, and documentation & 12 & 7 & 7 & 11 & 0.14 \\
\hline Medical-legal concerns & 12 & 7 & 9 & 9 & 0.42 \\
\hline Affordable Care Act & 17 & 2 & 14 & 4 & 0.34 \\
\hline Types of malpractice insurance & 17 & 2 & 13 & 5 & 0.18 \\
\hline Financial planning & 17 & 2 & 13 & 5 & 0.18 \\
\hline Disability insurance & 18 & 1 & 14 & 4 & 0.13 \\
\hline
\end{tabular}


Table 3 Comparison of affirmative candidate member and program director experience of mentorship and job preparation

\begin{tabular}{llcc}
\hline Item & Trainee & Program Director & $X^{2}$ p-value \\
\hline Faculty mentor or program director review CV & $74 \%$ & $72 \%$ & 0.88 \\
Faculty mentor or program director discuss career goals & $72 \%$ & $94 \%$ & $78 \%$ \\
Program director recommends where to apply for job & $64 \%$ & $50 \%$ & 0.05 \\
Faculty mentor or program director assist in contract negotiation & $26 \%$ & $70 \%$ & $94 \%$ \\
Fellows encouraged to review contracts with a lawyer & 70.06 \\
\hline
\end{tabular}

surgery program directors found that $87 \%$ agreed trainees should receive education in practice management [4]. Despite this, 34\% of programs offered no training in the business of medicine, and $70 \%$ indicated that their residents were inadequately trained.

There are limited data on the importance of education in the more traditionally academic components of employment, such as preparation for protocol development and teaching. A survey of maternal fetal medicine fellows demonstrated that the percentage of respondents who felt they had adequate training to apply for grants was $20 \%$ [5], which is not dissimilar from the $26 \%$ reported here. However, assessments of other key components of an academic practice such as teaching and protocol development are lacking in the post-graduate medical literature. Multiple studies have highlighted the importance of mentorship, which likely serves as a surrogate for roles in university-based medicine. In both gynecologic oncology and maternal fetal medicine fellowship, having a close research mentor has been associated with completion of the thesis, fellowship satisfaction, and productivity $[5,6]$. Having a faculty advisor or mentor has also been associated with a fellow's desire to enter academic practice. In our study, the similarity in responses to questions between program directors and fellows regarding mentorship may suggest a close relationship between trainee and educator, though response bias cannot be ruled out as a factor in these findings. However, the discrepancy between the two groups regarding a discussion of career goals is striking. Faculty advisors and program directors may, therefore, need to be more explicit or transparent about their intentions for career counseling with trainees.

The amount of time dedicated to certain topics varies by program, and it is apparent that program directors have different opinions about the need for time spent to achieve competency. This is true of surgical training programs, as well, and is apparent in the diverse approach to education in the practice of non-clinical medicine. A number of strategies have been proposed to increase competency with contract negotiation, coding compliance, and financial planning. Jones, et al. [7] reported on a 10-lecture curriculum in managed care and coding compliance given over two years to surgical residents. Its efficacy was impressive; surgical coding compliance increased nearly $50 \%$ over a 12-month period. Other approaches have included weekend retreats and mock trial presentations, all of which demonstrated significant improvement in measured knowledge categories and high participant satisfaction $[8,9]$.

There are several weaknesses to this study. The survey that was released was developed de novo, and is not validated, so its reproducibility may be in question. The response rates of $28 \%$ and $40 \%$ for candidate members and fellowship directors, respectively, are low, but are in line with other studies requiring physician response to survey invitations. There is also a likely component of bias, as more than two-thirds of candidate respondents had less than three years of experience, and about 90\% of had a university appointment or were in a community/academic hybrid practice. Relative overrepresentation of this population may skew perceptions on the need for additional academic-based professional training, but demonstrates that there are clearly concerns regarding tools for success as an academic gynecologic oncologist. Additionally, candidates were not queried about advanced training in research, business, or administration, which may have influenced their responses. Despite these limitations, this is the first study within the gynecologic oncology subspecialty to evaluate perceived preparation for measures outside of the usually studied surgical and medical oncology realm, and as such provides new insight into areas in which changes in didactic programs may be made. Larger investigations across multiple subspecialties, both on the residency and fellowship level, may assist in clarifying perceived additional educational need, with consideration being given to including such training as a core measure for trainee professionalism.

At our institution, we have initiated a more comprehensive program of didactics based on the current findings, now including lectures from risk management, billing and coding, and the institutional review board. Our approach, however, may not be universally applicable to all gynecologic oncology programs. In fact, since preparation in the business and academic practice of medicine is not a component of any core competency for training, creativity in curricula development should be encouraged. It is clear, though, that new graduates from gynecologic oncology 
fellowships feel inadequately prepared for some of the responsibilities they will have post-training. It remains incumbent on those entrusted to educate them to continuously strive to improve their programs and maintain open communication with the fellows so that a truly collaborative teaching environment can be developed.

\section{Conclusion}

Recent gynecologic oncology fellowship graduates want more training in the non-clinical, academic and business practice of medicine. Modifying curricula to more effectively prepare graduates for professional lives should be considered. Improved dialogue between trainer and trainee will be a crucial component of such a curricular evolution.

\section{Additional files}

Additional file 1: Candidate Member Survey. (DOCX $16 \mathrm{~kb}$ )

Additional file 2: Fellowship Program Director Survey. (DOCX 14 kb)

\section{Abbreviations}

ABOG: American Board of Obstetrics and Gynecology; ACGME: Accreditation Council for Graduate Medical Education; LOI: Letters of Intent; SGO: Society of Gynecologic Oncology

\section{Acknowledgements}

The authors would like to acknowledge the support and feedback from the Division of Gynecologic Oncology at the Sylvester Comprehensive Cancer Center, University of Miami.

\section{Funding}

Funding in part provided by Sylvester Comprehensive Cancer Center.

\section{Availability of data and materials}

Please contact author for data requests.

\section{Authors' contributions}

MS conceived the study, drafted the protocol, designed the questionnaires, performed the data analysis, and was primarily responsible for writing the manuscript. JS collected data, assisted in data analysis, created figures and tables, and assisted in writing the manuscript. GM participated in data collection and analysis. MH participated in study design and writing the manuscript. BS participated in conception of the study and writing the manuscript. All authors read and approved the final manuscript.

\section{Author information}

Dr. Schlumbrecht is an Associate Professor of Clinical Obstetrics and Gynecology in the Division of Gynecologic Oncology at Sylvester Comprehensive Cancer Center at the University of Miami. He serves as the Associate Director for the Gynecologic Oncology Fellowship Program and the Co-Director of Cancer Control and Prevention for Gynecologic Oncology.

\section{Ethics approval and consent to participate}

This research protocol was approved by the Institutional Review Board at the University of Miami (approved protocol 2016-0843).

\section{Consent for publication}

Not applicable.

\section{Competing interests}

The authors declare that they have no competing interests.

\section{Publisher's Note}

Springer Nature remains neutral with regard to jurisdictional claims in published maps and institutional affiliations.

\section{Author details}

'Division of Gynecologic Oncology, Sylvester Comprehensive Cancer Center, University of Miami, 1121 NW 14th St, Suite 345C, Miami, FL 33136, USA.

${ }^{2}$ Department of Obstetrics and Gynecology, University of Miami, Miami, USA.

Received: 3 August 2017 Accepted: 18 September 2017

Published online: 22 September 2017

\section{References}

1. Doo D, et al. Preparedness of Ob/Gyn residents for fellowship training in gynecologic oncology. Gynecol Oncol Rep. 2015;12:55-60.

2. Scribner DJ, Baldwin J, Mannel R. Gynecologic oncologists' perceptions of fellowship training. J Reprod Med. 2005;50(1):29-34.

3. Fakhry $\mathrm{S}$, et al. Surgical residents' knowledge of documentation and coding for professional services: an opportunity for focused educational offering. Am J Surg. 2007;194:236-67.

4. Lusco V, Martinez S, Polk H. Program directors in surgery agree that residents should be formally trained in business and practice management Am J Surg. 2005;189:11-3.

5. Sciscione A, Colmorgen G, D'Alton M. Factors affecting fellowship satisfaction, thesis completion, and career direction among maternal-fetal medicine fellows. Obstet Gynecol. 1998;91(6):1023-6.

6. Ramondetta L, et al. Mentorship and productivity among gynecologic oncology fellows. J Cancer Educ. 2003;18(1):15-9.

7. Jones $\mathrm{K}$, et al. Practice management education during surgical residency. Am J Surg. 2008;196:878-82.

8. Holak E, Kaslow O, Pagel P. Facilitating the transition to practice: a weekend retreat curriculum for business-of-medicine education of United States anesthesiology residents. J Anesth. 2010:24:807-10.

9. Drukteinis $D$, et al. Preparing emergency physicians for malpractice litigation: a joint emergency medicine residency-law school mock trial competition. J Emerg Med. 2014;46(1):95-103.
Submit your next manuscript to BioMed Central and we will help you at every step:

- We accept pre-submission inquiries

- Our selector tool helps you to find the most relevant journal

- We provide round the clock customer support

- Convenient online submission

- Thorough peer review

- Inclusion in PubMed and all major indexing services

- Maximum visibility for your research

Submit your manuscript at www.biomedcentral.com/submit
Biomed Central 